\title{
A Structure-Activity Relationship for Induction of Meningeal Inflammation by Muramyl Peptides
}

\author{
Margaret Burroughs, Eva Rozdzinski, Sibyl Geelen, and Elaine Tuomanen \\ Laboratory of Molecular Infectious Diseases, The Rockefeller University, New York, 10021
}

\begin{abstract}
Components of bacterial peptidoglycans have potent biological activities, including adjuvant effects, cytotoxicity, and induction of sleep. Mixtures of peptidoglycan components also induce inflammation in the lung, subarachnoid space, and joint, but the structural requirements for activity are unknown. Using a rabbit model for meningitis, we determined the biological activities of 14 individual muramyl peptides constituting $>90 \%$ of the peptidoglycan of the gram-negative pediatric pathogen Haemophilus influenzae. Upon intracisternal inoculation, most of the muropeptides induced leukocytosis in cerebrospinal fluid (CSF), influx of protein into CSF, or brain edema, alone or in combination. The disaccharide-tetrapeptide, the major component of all gram-negative peptidoglycans, induced CSF leukocytosis and protein influx at doses as low as $0.4 \mu \mathrm{g}(0.42 \mathrm{nM})$. Modification of the $\boldsymbol{N}$-acetyl muramic acid or substitution of the alanine at position four in the peptide side chain decreased leukocytosis but enhanced brain edema. As the size of the muropeptide increased, the inflammatory activity decreased. Muropeptides carrying the diaminopimelyl-diaminopimelic acid cross-link specifically induced cytotoxic brain edema. These findings significantly expand the spectrum of biological activities of natural muramyl peptides and provide the basis for a structure-activity relationship for the inflammatory properties of bacterial muropeptides. (J. Clin. Invest. 1993. 92:297-302.) Key words: meningitis • peptidoglycan • central nervous system inflammation • blood brain barrier permeability • cell wall
\end{abstract}

\section{Introduction}

Under the age of 5 years, bacterial meningitis is most commonly caused by Haemophilus influenzae, Streptococcus pneumoniae, or Neisseria meningitidis. In all three instances, antibiotic-induced bacterial killing leads to the rapid release of bacterial components into the environment of the host and strongly enhances the inflammatory response and tissue damage $(1,2)$. It is generally accepted that this inflammatory response is directed at endotoxin in the case of gram-negative pathogens and at cell wall components, such as peptidoglycan and teichoic

This material was presented in part at the International Conference for Antimicrobials and Chemotherapy, October 1992, Anaheim, CA.

Address correspondence to Dr. Elaine I. Tuomanen, Laboratory of Molecular Infectious Diseases, The Rockefeller University, 1230 York Avenue, New York, NY 10021. Dr. Geelen's present address is Department of Infectious Diseases, University Children's Hospital "Het Wilhelmina Kinderziekenhuis," Utrecht, The Netherlands.

Received for publication 19 October 1992 and in revised form 22 February 1993.

J. Clin. Invest.

(c) The American Society for Clinical Investigation, Inc.

0021-9738/93/07/0297/06 \$2.00

Volume 92, July 1993, 297-302 acid, in the case of the pneumococcus $(1,3,4)$. More recently, significant inflammatory activity has also been ascribed to the purified peptidoglycan of gram-negative bacteria, including $H$. influenzae $(2,5)$. For both gram-positive and gram-negative pathogens, these inflammatory peptidoglycans are mixtures of numerous complex muropeptides. The ability of individual muropeptides to incite the various aspects of inflammation in either gram-positive or gram-negative disease is undefined. Having recently determined the complete structure of the peptidoglycan of $H$. influenzae (6), we sought to generate the first structure-activity profile for the inflammatory activities of individual muropeptides comprising a complete bacterial peptidoglycan.

The gram-negative cell wall is composed of a peptidoglycan backbone of repeating $N$-acetylglucosamine- $N$-acetylmuramic acid units linked by a family of over 15 different types of peptide side chains $(6,7)$. Each bacterial species has a unique pattern of amino acids in the peptide side chains. Gram negative peptidoglycan is further decorated by covalently bound lipoprotein and noncovalently bound peptidoglycan-associated proteins (8). The insoluble cell wall macromolecule is assembled and continuously manicured by a set of enzymes that are the targets of the $\beta$-lactam family of antibiotics. The products of this dynamic process, the muropeptides, are released into the environment during growth and antibiotic-induced bacterial killing $(2,9)$. Thus, both the intact cell wall macromolecule and its soluble muropeptides are potential inflammatory mediators.

Bacterial cell wall and its muropeptide subcomponents have potent activities in biological systems. The adjuvant effect of muramyl dipeptide is well characterized (10). The 1,6-anhydro-muramyl tetrapeptide has been shown to promote slow wave sleep, kill ciliated cells, and promote arthropathic changes (11-15). We have shown that $H$. influenzae cell wall, purified peptidoglycan, or a mixture of soluble muropeptides incite inflammation in the subarachnoid space of rabbits $(2$, $5)$. These components have a particularly high specific activity for the production of brain edema. The inflammatory activity of these muropeptides appears to be sensitive to structural modifications, since altered muropeptides characteristic of peptidoglycan from ampicillin resistant $H$. influenzae have enhanced inflammatory bioactivities (2). These observations suggest that individual muropeptides might provoke some or all aspects of inflammation in the subarachnoid space.

Definitive structural assignment has been ascertained for 17 individual muropeptides from $H$. influenzae, representing $96 \%$ of the total peptidoglycan (6). The major monomer, a disaccharide tetrapeptide ( Tetra) composed of $N$-acetylglucosamine- $N$-acetylmuramic acid with a peptide side chain of AlaGlu-Dap-Ala, represents more than $30 \%$ of the bacterial peptidoglycan; $27 \%$ of the peptidoglycan is a dimeric form of the same molecule. Variations of these two structures include 1,6anhydro-muramyl derivatives, amino acid substitutions for the 
Table I. Ability of Individual Muropeptides to Provoke Meningeal Inflammation

\begin{tabular}{|c|c|c|c|c|c|}
\hline Muropeptide & Structure & Dose* & Leukocytes $^{\ddagger}$ & Protein influx $x^{9}$ & Brain edema" \\
\hline Control & & & $80 \pm 12$ & $0.01 \pm 0.12$ & $392 \pm 5$ \\
\hline Tri & GM-Ala-Glu-Dap & 4.0 & $37 \pm 19$ & $0.55 \pm 0.2^{1}$ & $396 \pm 5$ \\
\hline Tetra & GM-Ala-Glu-Dap-Ala & 35.0 & $662 \pm 245^{1}$ & $0.87 \pm 0.43^{\prime}$ & $387 \pm 9$ \\
\hline Tetra $^{\text {nr }}$ & GM $^{\mathrm{nr}}$-Ala-Glu-Dap-Ala & 1.0 & $126 \pm 73$ & $0.03 \pm 0.01$ & $392 \pm 7$ \\
\hline Tetra (A/S) & GM-Ala-Glu-Dap-Asp/Ser & 1.5 & $160 \pm 97$ & $0.38 \pm 0.25^{1}$ & $399 \pm 3^{1}$ \\
\hline Tetra (Gly) & GM-Ala-Glu-Dap-Gly & 3.0 & $126 \pm 117$ & $0.55 \pm 0.15^{1}$ & $385 \pm 8$ \\
\hline Tetra-tri & $\begin{array}{l}\text { GM-Ala-Glu-Dap } \\
\qquad \begin{array}{l}\text { Ala-Dap-Glu-Ala-GM }\end{array}\end{array}$ & 6.0 & $621 \pm 390^{\prime}$ & $0.81 \pm 0.19^{\prime}$ & $402 \pm 4^{1}$ \\
\hline Tetra-tri (Anh) & $\begin{array}{l}\text { GM-Ala-Glu-Dap } \\
\qquad \begin{array}{l}\text { Ala-Dap-Glu-Ala-GM (Anh) }\end{array}\end{array}$ & 1.5 & $56 \pm 37$ & $0.33 \pm 0.31^{1}$ & $394 \pm 8$ \\
\hline Tetra-tetra & $\begin{array}{l}\text { GM-Ala-Glu-Dap-Ala } \\
\qquad \begin{array}{l}\text { Ala-Dap-Glu-Ala-GM }\end{array}\end{array}$ & 20.0 & $145 \pm 120$ & $0.38 \pm 0.2^{1}$ & $390 \pm 7$ \\
\hline Tetra-Tetra (Gly) & $\begin{array}{l}\text { GM-Ala-Glu-Dap-Gly } \\
\qquad \begin{array}{l}\text { | Ala-Dap-Glu-Ala-GM } \\
\text { A }\end{array}\end{array}$ & 2.0 & $20 \pm 8$ & $0.07 \pm 0.02$ & $390 \pm 8$ \\
\hline Tetra-tetra (Anh) & $\begin{array}{l}\text { GM-Ala-Glu-Dap-Ala } \\
\qquad \begin{array}{l}\text { Ala-Dap-Glu-Ala-GM (Anh) }\end{array}\end{array}$ & 4.0 & $64 \pm 55$ & $0.78 \pm 0.26^{\prime}$ & $391 \pm 8$ \\
\hline $\begin{array}{l}\text { Tetra-tetra } \\
\text { (dap-dap) }\end{array}$ & $\begin{array}{l}\text { GM-Ala-Glu-Dap-Ala } \\
\text { | } \\
\text { Ala-Dap-Glu-Ala-GM }\end{array}$ & 2.0 & $85 \pm 26$ & $0.17 \pm 0.14$ & $398 \pm 1^{1}$ \\
\hline $\begin{array}{r}\text { Tetra-tetra }^{\text {nr }} \\
\text { (dap-dap) }\end{array}$ & $\begin{array}{l}\text { GM-Ala-Glu-Dap-Ala } \\
\text { I } \\
\text { Ala-Dap-Glu-Ala-GM }{ }^{\mathrm{nr}}\end{array}$ & 1.0 & $83 \pm 8$ & $0.04 \pm 0.02$ & $404 \pm 2^{5}$ \\
\hline Tetra-tetra-tetra & $\begin{array}{l}\text { GM-Ala-Glu-Dap-Ala } \\
\text { Ala-Dap-Glu-Ala-GM } \\
\text { GM-Ala-Glu-Dap-Ala }\end{array}$ & 2 & $34 \pm 20$ & $1.2 \pm 0.20^{\prime}$ & $384 \pm 9$ \\
\hline $\begin{array}{l}\text { Tetra-tetra-tetra } \\
\text { (Anh) }\end{array}$ & $\begin{array}{l}\text { GM-Ala-Glu-Dap-Ala } \\
\text { Ala-Dap-Glu-Ala-GM } \\
\text { GM(Anh)-Ala-Glu-Dap-Ala }\end{array}$ & 1.0 & $36 \pm 6$ & $0.40 \pm 0.14^{\top}$ & $391 \pm 8$ \\
\hline
\end{tabular}

\footnotetext{
* $\mu \mathrm{g}$ of muropeptide found in $100 \mu \mathrm{g}$ of peptidoglycan. ${ }^{\ddagger}$ Mean $\pm \mathrm{SD}$ expressed in cells $/ \mu \mathrm{l} \mathrm{CSF}$ at $6 \mathrm{~h} .{ }^{8} \mathrm{mg}$ protein $/ \mathrm{ml} \mathrm{CSF}(\mathrm{mean} \pm \mathrm{SD}$ ) accumulated from 0 to $6 \mathrm{~h}$. "Mean $\pm \mathrm{SD}$ of values expressed as $\mathrm{g}_{2} \mathrm{O} / 100 \mathrm{~g}$ dry brain wt at $6 \mathrm{~h}$. ' $P<0.001$ compared to control value.

${ }^{\mathrm{nr}}$ Nonreduced muramic acid.
}

alanine in position four of the peptide chain, or truncation of this alanine. In this report, we assign specific bioactivities to over a dozen individual muramyl peptides derived from a gram-negative peptidoglycan. We then derive a structure activity relationship for three parameters of meningeal inflammation: leukocytosis in cerebrospinal fluid (CSF), ${ }^{1}$ influx of protein into CSF, and brain edema.

\section{Methods}

\section{Experimental procedures}

Preparation of muropeptides. Muropeptides were prepared from strain $H$. influenzae MAP by an adaption of the method of Glauner $(7,16)$, as described in detail elsewhere (6). Briefly, crude cell wall from 10

1. Abbreviations used in this paper: Ala, alanine; CSF, cerebrospinal fluid; dap, diaminopimelic acid. liters of culture was precipitated in boiling $10 \%$ SDS, digested with alpha amylase (Sigma Chemical Co., St. Louis, MO) and pronase (Calbiochem-Novabiochem Corp., La Jolla, CA) and reprecipitated in boiling SDS. The purified protein-free peptidoglycan was digested with Streptomyces globisporus muramidase (Miles Scientific, Naperville, IL), reduced with sodium borohydride, and subjected to reverse phase HPLC at room temperature using a gradient from 0 to $15 \%$ methanol in phosphate buffer under established conditions (17). Individual muropeptides were desalted by HPLC using a gradient from 0 to $50 \%$ acetonitrile in $0.05 \%$ trifluoroacetic acid/water and evaporated until dry. The structural assignments of the individual muropeptides have been previously determined by fast atom bombardment mass spectrometry/tandem mass spectrometry, and amino acid analysis ( $\mathrm{Ta}$ ble I) (6).

Rabbit model for meningitis. The rabbit model was performed according to an established protocol $(3,18)$. Specific pathogen-free, New Zealand white rabbits ( $2 \mathrm{~kg}$; Hare Marland, Nutley, $\mathrm{NJ}$ ) were anesthetized with Valium ( $2.5 \mathrm{mg} / \mathrm{kg}$, s.c.; Hoffmann-La Roche Inc., Nutley, 
$\mathrm{NJ}$ ), ketamine ( $35 \mathrm{mg} / \mathrm{kg}$, i.m.; Aveco, Fort Dodge, IA), and xylazine $(5 \mathrm{mg} / \mathrm{kg}$, i.m.; Miles Laboratories, Shawnee, KS), and a helmet of dental acrylic was affixed to the calvarium. $24 \mathrm{~h}$ later, the rabbits were anesthetized with ethyl carbamate $(1.75 \mathrm{~g} / \mathrm{kg}$; Aldrich Chemical Co., Milwaukee, WI ) and pentobarbital ( $15 \mathrm{mg} / \mathrm{kg}$; Abbott Laboratories, Abbott Park, IL) and placed in a stereotaxic frame. A spinal needle was introduced into the cisterna magna and $300 \mu \mathrm{l}$ of CSF was withdrawn.

For injection into rabbits, lyophilized, individual muropeptides were resuspended in pyrogen-free water and adjusted to the desired concentration in a volume of $200 \mu \mathrm{l}$. Each muropeptide was initially injected intracisternally into four rabbits at a dose corresponding to its relative representation in $100 \mu \mathrm{g}$ of cell wall (Table I). This dose was chosen since it induces a strong inflammatory response in this model and a mixture of the individual muropeptides derived from $100 \mu \mathrm{g}$ of intact wall has been shown to generate a similar inflammatory response upon injection into the subarachnoid space (2). The monomer tetrapeptide was further tested over a concentration range of 35 to $0.04 \mu \mathrm{g}$ ( 32 to $0.04 \mathrm{nM}$ ). To allow comparison of specific activities between components, all muropeptides were also tested on an equimolar basis $(0.3$ to $0.4 \mathrm{nM})$. For five control animals, desalting buffer was evaporated until dry, resuspended in water, and administered in a volume of $200 \mu \mathrm{l}$.

Samples of CSF were withdrawn at 2, 4, and $6 \mathrm{~h}$ after inoculation of the muropeptide into the subarachnoid space. At $6 \mathrm{~h}$, the rabbits were killed with an overdose of pentobarbital, and the brains were harvested to measure brain edema by comparing wet to dry wt $(19,20)$. The brains were weighed, dried at $100^{\circ} \mathrm{C}$ for $7 \mathrm{~d}$ (the nadir of weight for control animals), and weighed once more. Brain edema was calculated using an established formula $(19,20)$. For all CSF samples, leukocyte density was measured using a counter (Coulter Electronics Inc., Hialeah, FL). CSF samples were centrifuged at $10,000 \mathrm{~g}$ for $5 \mathrm{~min}$ and the supernatant was stored at $-70^{\circ} \mathrm{C}$ until assayed for protein concentration using the bicinchoninic acid method (BCA Kit; Pierce Chemical Co., Rockford, IL). Significant differences in the means of these determinations in groups of rabbits were determined using the Student's $t$ test.

\section{Results}

The ability of each of 14 muropeptides to induce leukocytosis, blood-brain barrier permeability, and brain edema upon intracisternal inoculation was tested. To maintain physiological relevance, the initial characterization was carried out at a dose of the muropeptide that represented the amount of the individual component found in $100 \mu \mathrm{g}$ of peptidoglycan, an amount previously documented as inflammatory. All but two of the muropeptides were active for one or more parameters of inflammation (Table I). The nonreduced monomeric tetrapeptide $\left(\right.$ Tetra $\left.^{\mathrm{nr}}\right)$ and the dimeric Tetra-tetra(gly) were inactive. Only two muropeptides induced leukocytosis, the major monomeric tetrapeptide (Tetra) and the Tetra-tri dimer. In the case of the Tetra, this activity showed a concentration optimum of $4 \mu \mathrm{g}$ (Fig. 1, upper graph); higher doses produced accumulation of protein in CSF without attendant leukocytosis (Fig. 1, lower graph). Leukocytosis was always accompanied by protein influx, a finding consistent with injury to the blood-brain barrier associated with leukocyte recruitment.

Most of the muropeptides induced only influx of protein into CSF. Included in this group were variants of the Tetra monomer: Tri, Tetra (Gly), and the oligomeric muropeptides: Tetra-tri (Anh), Tetra-tetra, Tetra-tetra (Anh), Tetra-tetratetra, and Tetra-tetra-tetra (Anh). Two unusual Tetra-tetra dimers containing diaminopimelyl-diaminopimelic acid (dapdap) crosslinks produced only brain edema. One muropeptide produced a combination of the two inflammatory parameters:
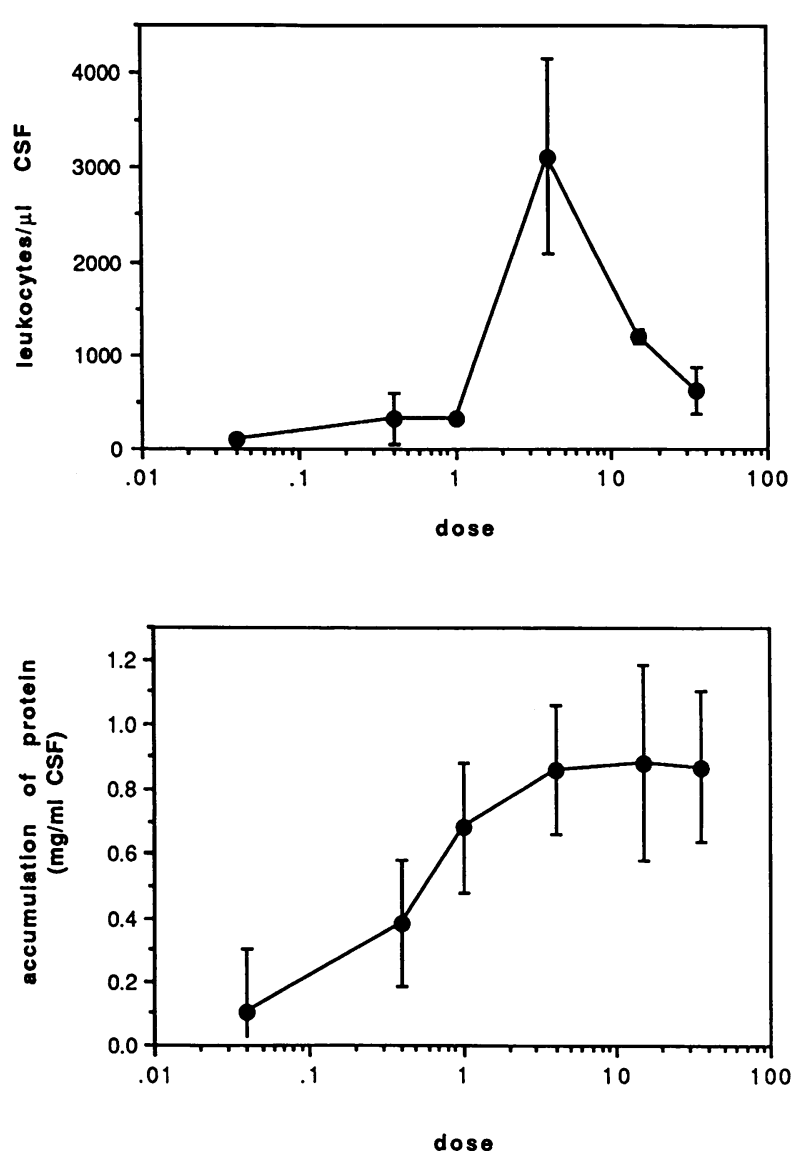

Figure 1. Effect of dose of Tetra on the ability to induce meningeal inflammation at $6 \mathrm{~h}$. Upper panel: leukocytosis (mean $\pm \mathrm{SD})$; lower panel: protein accumulation in CSF ( mean $\pm S D)$ from time 0 to $6 \mathrm{~h}$.

Tetra (A/S). These individual activities indicate that a mixture of these components in proportions relevant to the composition of the total cell wall would be expected to induce all three of the cardinal features of meningeal inflammation.

To determine the structural features of the individual glycopeptides that determined biological activity, families of muropeptides were compared at equimolar concentrations. Fig. 2 illustrates the strong influence of the D-alanine in position four of the peptide side chain on bioactivity. Only the major monomer Tetra produced leukocytosis at $4 \mathrm{nM}$ and this activity was lost at $0.4 \mathrm{nM}$. All monomers induced approximately equivalent protein influx at $4 \mathrm{nM}$ but substitution of the terminal alanine by aspartic acid, serine, or glycine caused a significantly lower activity at $0.4 \mathrm{nM}$. In contrast, cleavage of the alanine to create a tripeptide (Tri) resulted in greater protein influx at the lower dose of $0.4 \mathrm{nM}$. Brain edema was not a strong effect of these monomeric muropeptides (Fig. 2). However, the dose of these monomeric muropeptides that produced the greatest protein influx (the tripeptide: $0.4 \mathrm{nM}$ and Tetra Asp/Ser: $4 \mathrm{nM}$ ) produced brain edema above the maximum of controls.

Multimeric forms of the Tetra monomer, the Tetra-tetra and Tetra-tetra-tetra muropeptides, failed to induce protein influx when compared to the monomer at $0.4 \mathrm{nM}$ (Table II). Loss of biological activity was also seen upon covalent linkage of the Tetra and Tri active species to a Tetra-tri dimer. However, not all oligomers were inactive. The dimers containing the diaminopimelyl-diaminopimelic acid cross-link were po- 

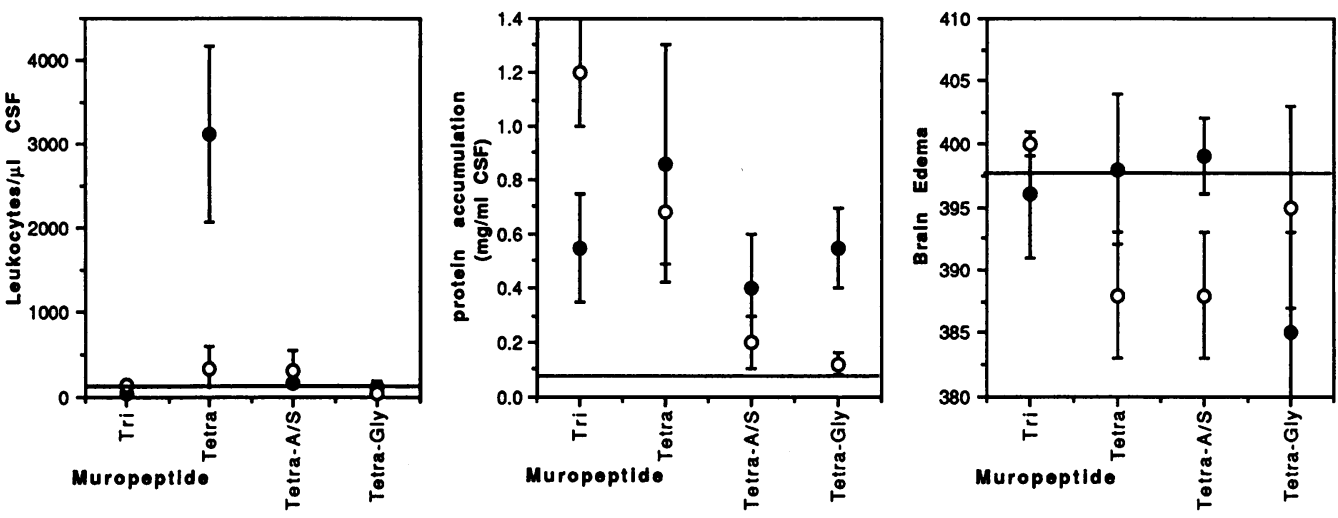

Figure 2. Influence of variations in the peptide side chain on the bioactivity of monomeric muropeptides. Each muropeptide was injected at doses of $4.0(\bullet)$ and $0.4 \mathrm{nM}$ (O) into four animals each and CSF cytochemical parameters were determined at $6 \mathrm{~h}$. Abbreviations are as in Table I. (Left) Leukocytosis $($ mean $\pm \mathrm{SD}) ;($ Middle) amount of protein accumulated in CSF over $6 \mathrm{~h}$ (mean \pm SD); (Right) brain

edema (mean $\pm \mathrm{SD}^{\circ}$ of $\mathrm{g}$ water per $100 \mathrm{~g}$ of dry brain $\mathrm{wt}$ ). Maximum of control values (bar) is derived from five animals receiving lyophilized HPLC desalting buffer alone. All mean values which fall above the bars are significantly different from controls at $P<01$. In the middle panel, all values at $4 \mathrm{nM}$ are not significantly different, while the values for Tri, Tetra A/S, and Tetra Gly are significantly different $(P<0.01)$ from Tetra at the $0.4 \mathrm{nM}$ dose.

tent inducers of brain edema (Table $\mathrm{I} ; 0.5 \mathrm{nM}$ ). This indicated that the involvement of the D-alanine in a cross-link to a neighboring peptide side chain inhibited bioactivity while the dapdap cross-link created a uniquely cytotoxic species.

Alterations in the structure of the glycan backbone also affected the bioactivities of the muropeptides in the subarachnoid space. The ability to induce leukocytosis or brain edema was sensitive to the state of reduction or hydration of the $N$ acetyl muramic acid at positions one and six. When compared at $0.5 \mathrm{nM}$, the nonreduced disaccharide tetrapeptide ( Tetra ${ }^{\mathrm{nr}}$ ) produced brain edema ( $398 \pm 1 \mathrm{~g}$ water $/ 100 \mathrm{~g}$ of dry brain wt) while the fully reduced Tetra did not $(388 \pm 5 \mathrm{~g}$ water $/ 100 \mathrm{~g}$ of dry brain wt $)(P<0.01)$. The nonreduced Tetra-tetra dimer also showed a slightly greater ability than the reduced dimer to cause brain edema ( $404 \pm 1$ vs. $398 \pm 2 \mathrm{~g}$ water $/ 100 \mathrm{~g}$ of dry brain wt). Dehydration to create a 1,6-anhydro linkage on the dimer imparted the ability to produce protein influx: when compared at $0.2 \mathrm{nM}$, Tetra-tetra produced no influx $(0.02 \pm 0.2$ $\mathrm{mg} / \mathrm{ml}$ ) while the Tetra-tetra(Anh) produced $0.3 \pm 0.08 \mathrm{mg} /$ $\mathrm{ml}(P<0.01)$. Thus, changes in the glycan backbone enhanced the incidence and degree of brain edema and protein influx.

\section{Discussion}

The major muropeptides from the peptidoglycan of $H$. influen$z a e$ are representative of the peptidoglycan components of most gram-negative pathogens $(6,7)$. When challenged with mixtures of these muropeptides intracisternally, animals develop signs and symptoms that mimic natural meningitis, including CSF leukocytosis, increased blood-brain barrier permeability, and brain edema $(2,5,21)$. This is similar to the inflammatory response evoked by mixtures of gram-positive peptidoglycan components that contain similar glycopeptides (with lysine substituted for diaminopimelic acid) as well as teichoicated species $(3,4)$. When released through cell wall turnover or during antibiotic-induced autolysis, muropeptides constitute a library of biologically active molecules that contribute to the pathophysiology of bacterial meningitis. Three parameters of inflammation characteristic of meningitis were studied here. The ability to induce leukocytosis in CSF implies induction of changes in vascular endothelial cells so as to recruit leukocytes across the blood-brain barrier in a CD18-de- pendent manner (22). Accumulation of protein in CSF is regarded as a marker of enhanced blood-brain barrier permeability, which can be increased sufficiently so as to lead to vasogenic brain edema. Alternatively, brain edema occurring in the absence of enhanced vascular endothelial permeability indicates cytotoxic effects directly on neuronal or glial cells. Natural gram-negative muramyl peptides were able to profoundly derange each of these parameters of inflammation and thereby constitute a family of biological effector molecules with potent activities in the subarachnoid space. These activities significantly expand the biological properties ascribed to muramyl peptides.

The most abundant species in all gram-negative peptidoglycans, the monomeric disaccharide tetrapeptide (Tetra), displayed the highest specific activity for leukocytosis, suggesting that its abundant release during cell wall turnover and bacterial killing would be sufficient to incite inflammatory changes. The only other muropeptide to induce leukocytosis was the Tetratri component. Previous studies have shown that the bioactivity of peptidoglycan derived from ampicillin resistant $\mathrm{H}$. influenzae is greater than that of sensitive strains and that this property co-transforms with changes in peptidoglycan structure $(2,6)$. This enhanced activity can now be ascribed, at least in part, to the greater amount of the proinflammatory Tetra-tri muropeptide present in the peptidoglycan of resistant strains.

The ability of the Tetra muropeptide to induce leukocytosis and the Tri to induce protein influx did not increase in a linear

Table II. Comparison of the Influence of Muropeptide Size on Inflammatory Activity in the Subarachnoid Space

\begin{tabular}{lccc}
\hline \multicolumn{1}{c}{ Muropeptide } & Leukocytosis* $^{*}$ & Protein influx & Brain edema \\
\hline Tetra & $322 \pm 169$ & $0.68 \pm 0.2$ & $388 \pm 5$ \\
Tetra-tetra & $80 \pm 41^{\ddagger}$ & $0.20 \pm 0.2^{\ddagger}$ & $386 \pm 6$ \\
Tetra-tetra-tetra & $19 \pm 8^{\ddagger}$ & $0.10 \pm 0.1^{\ddagger}$ & $394 \pm 4$ \\
Tri & $137 \pm 53$ & $1.21 \pm 0.2$ & $399 \pm 1$ \\
Tetra-tri & $57 \pm 37^{\ddagger \S}$ & $0.19 \pm 0.2^{\ddagger \S}$ & $387 \pm 7^{\S}$ \\
\hline
\end{tabular}

* Units are as defined in Table I. All comparisons were made upon injection of $0.3-0.4 \mathrm{nM}$ of muropeptide. ${ }^{ \pm} P<0.001$ vs. Tetra. ${ }^{8} P$ $<0.001$ vs. Tri. 
fashion with increasing dose, indicating a concentration optimum exists for some inflammatory properties of muropeptides. This is not exhibited by all parameters of inflammation for a given muropeptide, in that protein influx induced by the Tetra followed a more conventional linear curve with dose. This suggests that it is not the physical state of the muropeptide that is altered with concentration, as has been shown for lipidcontaining molecules such as endotoxin. At this time, no explanation for this behavior is apparent. One hypothesis is based on the observation that pneumococcal cell wall binds to a receptor on endothelial cells, which triggers production of an inhibitory substance from the eukaryotic cells, which prevents further attachment of bacteria (23). It is conceivable that as the dose of some muropeptides increases, the production of an antagonist from the eukaryotic cells may be accelerated and thereby may inhibit the net inflammatory activity.

The proinflammatory activities of the muropeptides were highly dependent on variations in the structures of the individual components (Fig. 3). While most species induced influx of protein, the ability to induce leukocytosis and brain edema appeared to vary inversely. The presence of D-alanine at position four of the peptide side chain was required for induction of leukocytosis. Substitution by aspartic acid, serine, or glycine decreased leukocytosis and protein influx, while truncation of the tetrapeptide to a tripeptide enhanced protein influx and brain edema. Involvement of the alanine in a cross-link creating the major dimer and trimer also reduced bioactivity.

The third amino acid in the peptide chain, diaminopimelic acid, also appeared to be important to bioactivity, since engagement in a dap-dap cross-link characterized components producing only brain edema. The absence of concomitant protein influx suggests that these components induce cytotoxic rather than vasogenic brain edema. The number of dap-dap crosslinks is a highly regulated characteristic of peptidoglycan, being particularly increased in slowly growing and nongrowing $E$. coli (24). The slow rate of growth characteristic of pathogens in the nutrient-poor CSF indicates that these dap-dap-containing muropeptides may be important cytotoxins during meningitis. It should be noted that cytotoxicity for ciliated cells is decreased by dap-dap cross-linkage (12). In addition, the presence of lysine instead of diaminopimelic acid abolishes ciliated cell cytotoxicity (12) but it is known that mixtures of muropeptides from gram-positive bacteria that all contain lysine at position three are highly inflammatory in the subarachnoid space $(1,4)$. These differences indicate that important struc-

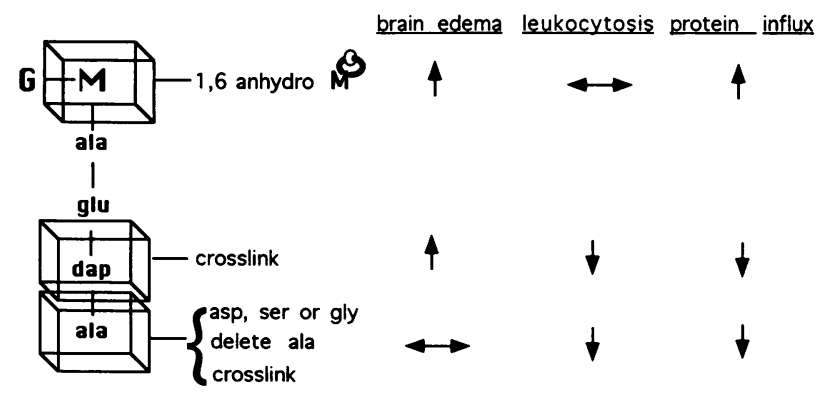

Figure 3. Schematic representation of the structure/activity relationship of muropeptides for the induction of brain edema, leukocytosis, and protein influx into CSF. Direction of arrow indicates increase, decrease, or no effect on activity.
Table III. Comparison of the Influence of Structural Variations Within Bacterial Muropeptides on Bioactivity

\begin{tabular}{llllll}
\hline & \multicolumn{4}{c}{ Site of variation } \\
\cline { 2 - 5 } \multicolumn{1}{c}{ Bioactivity } & lys vs. dap & Ala & $N$-acetylmuramic acid & Reference \\
\cline { 2 - 5 } \multicolumn{2}{c}{ Peptide chain } & & & this study \\
CSF leukocytosis & - & + & - & this study \\
CSF protein influx & - & + & + & this study \\
Brain edema & - & + & + & 10 \\
Ciliated cell toxicity & + & + & - & 11,12 \\
Sleep & - & - & + & + & 10 \\
Immunoadjuvant & - & - & & + & 8 \\
\hline
\end{tabular}

+ denotes that a change in this position affects the indicated bioactivity.

tural specificity exists between cytotoxic effects of muropeptides in different organs (Table III).

The 1,6-anhydro linkage is present in tracheal cytotoxin and the sleep peptide (12-14) and appears to activate muropeptides for the induction of vasogenic brain edema. All of these bioactivities share the common feature that they involve induction of IL-1 from the target cells $(13,25)$. This may suggest related ligand-receptor interactions for these diverse biological effects.

When the structural requirements for activity of muramyl peptides in several biological systems are compared, the pattern for induction of inflammatory parameters in brain appears to be unique ( Table III). The ability to induce leukocytosis was the most sensitive to any change in the peptide side chain. The ability to induce brain edema was activated by changes in peptide positions three or four and by reduction or 1,6-anhydro modification of the $N$-acetylmuramic acid. The ability to enhance vascular permeability as measured by protein influx into CSF was the single parameter studied that was least affected by structural variation. The presence of the dapdap link or increase in size of the muropeptide to a multimer consistently attenuated this activity.

This work provides the first detailed structure-activity profile for the bioactivities of the major components of gram-negative peptidoglycans in the subarachnoid space. The unique inflammatory profile of the individual muropeptides suggests that each muramyl peptide interacts with host defenses in a specific and individual manner. Based on the differential inflammatory properties defined here, the variation in the amounts and structures of the peptidoglycan subcomponents of each bacterial species can be predicted to produce different species-specific constellations of inflammatory effects. These are most readily apparent in the different patterns of inflammation caused by isogenic $\beta$ lactam-resistant vs. sensitive $H$. influenzae, which differ in their peptidoglycan composition by the amounts of two highly inflammatory muropeptides $(2,6)$. In this context, the individual muropeptides constitute a code for disease.

\section{Acknowledgments}

This work was supported by National Institute of Allergy and Infectious Diseases grant 27913 (to E. Tuomanen) and a Mary P. Dole Medical Fellowship (to M. Burroughs). 


\section{References}

1. Tuomanen, E., B. Hengstler, R. Rich, M. Bray, O. Zak, and A. Tomasz. 1987. Nonsteroidal anti-inflammatory agents in the therapy of experimental pneumococcal meningitis. J. Infect. Dis. 155:985-990.

2. Burroughs, M. H., S. Prasad, C. Cabellos, P. Mendelman, and E. Tuomanen. 1993. The biological activities of peptidoglycan in experimental Haemophilus influenzae meningitis. J. Infect. Dis. 167:464-8.

3. Tuomanen, E., H. Liu, B. Hengstler, O. Zak, and A. Tomasz. 1985. The induction of meningeal inflammation by components of the pneumococcal cell wall. J. Infect. Dis. 151:859-868.

4. Tomasz, A., and K. Saukkonen. 1989. The nature of cell wall-derived inflammatory components of pneumococci. Pediatr. Infect. Dis. J. 8:902-903.

5. Burroughs, M., C. Cabellos, S. Prasad, and E. Tuomanen. 1992. Bacteria components and the pathophysiology of injury to the blood-brain barrier: does cell wall add to the effects of endotoxin in gram-negative meningitis? $\mathrm{J}$. Infect. Dis. 165(Suppl. 1):S82-5.

6. Burroughs, M. H., Yoon, D. Gage, and E. I. Tuomanen. 1992. The composition of $H$. influenzae peptidoglycan. J. Biol. Chem. In press.

7. Glauner, B. 1988. Separation and quantification of muropeptides with high-performance liquid chromatography. Anal. Biochem. 172:451-464.

8. Ingraham, J. L., O. Maaloe, and F. C. Neidhardt. 1983. Growth of the Bacterial Cell. Sinauer Associates, Sunderland, MA. 435 pp.

9. Kitano, K., E. Tuomanen, and A. Tomasz. 1986. Transglycosylase and endopeptidase participate in the degradation of murein during autolysis of Escherichia coli. J. Bacteriol. 167:759-765.

10. Ellouz, F., R. Ciorbaru, and E. Lederer. 1974. Minimal structural requirements for adjuvant activity of bacterial peptidoglycan derivatives. Biochem. Biophys. Res. Commun. 59:1317-1325.

11. Fleming, T. J., D. E. Wallsmith, and R. S. Rosenthal. 1986. Arthropathic properties of gonococcal peptidoglycan fragments: implications for the pathogenesis of disseminated gonococcal disease. Infect. Immun. 52:600-608.

12. Goldman, W. E., D. G. Klapper, and J. B. Baseman. 1982. Detection, isolation, and analysis of a released Bordetella pertussis product toxic to cultured tracheal cells. Infect. Immun. 36:782-794.

13. Kreuger, J., D. Davenne, J. Walter, S. Shoham, S. Kubillus, R. Rosenthal, S. Martin, and K. Biemann. 1987. Bacterial peptidoglycans as modulators of sleep. Brain Res. 403:258-266.

14. Pappenheimer, J. R., G. Koski, V. Fencl, M. L. Karnovsky, and J.
Krueger. 1975. Extraction of sleep-promoting Factor $\mathbf{S}$ from cerebrospinal fluid and from brains of sleep-deprived animals. J. Neurophysiol. (Bethesda). 38:1299-1311.

15. Rosenthal, R. S., W. Nogami, B. T. Cookson, W. E. Goldman, and W. J. Folkening. 1987. Major fragment of soluble peptidoglycan released from growing Bordetella pertussis is tracheal cytotoxin. Infect. Immun. 55:2117-2120.

16. Glauner, B., and U. Schwarz. 1983. The analysis of murein composition with high-pressure-liquid chromatography. In The Target of Penicillin. R. Hackenbeck, editor. Walter de Gruyter \& Co, Berlin. 29-34.

17. Tuomanen, E., J. Schwartz, S. Sande, K. Light, and D. Gage. 1989. Unusual composition of peptidoglycan in Bordetella pertussis. J. Biol. Chem. 264:1 1093-11098.

18. Dacey, R. G., and M. A. Sande. 1974. Effect of probenecid on cerebrospinal fluid concentrations of penicillin and cephalosporin derivatives. Antimicrob. Agents Chemother. 6:437-441.

19. Tauber, M. G., A. M. Shibl, C. J. Hackbarth, J. W. Larrick, and M. A. Sande. 1987. Antibiotic therapy, endotoxin concentration in cerebrospinal fluid, and brain edema in experimental Escherichia coli meningitis in rabbits. J. Infect. Dis. 156:456-462.

20. Tauber, M. G., H. Khayam-Bashi, and M. A. Sande. 1985. Effects of ampicillin and corticosteroids on brain water content, cerebrospinal fluid pressure and cerebrospinal fluid lactate levels in experimental pneumococcal meningitis. J. Infect. Dis. 151:528-534.

21. Tuomanen, E., B. Hengstler, O. Zak, and A. Tomasz. 1987. Induction of meningeal inflammation by diverse bacterial cell walls. Eur. J. Clin. Microbiol. 5:682-684.

22. Tuomanen, E., K. Saukkonen, S. Sande, C. Cioffe, and S. D. Wright. 1989. Reduction of inflammation, tissue damage, and mortality in bacterial meningitis in rabbits treated with monoclonal antibodies against adhesion-promoting receptors of leukocytes. J. Exp. Med. 170:959-969.

23. Geelen, S., C. Battacharyya, and E. Tuomanen. 1993. Cell wall mediates pneumococcal attachment and cytopathology to human endothelial cells. Infect. Immun. 61:1538-1543.

24. Tuomanen, E., and R. Cozens. 1987. Changes in peptidoglycan composition and penicillin binding proteins in slowly growing Escherichia coli. J. Bacteriol. 169:5308-5310.

25. Saukkonen, K., C. Cioffe, S. Wolpe, B. Sherry, A. Cerami, and E. Tuomanen. 1990. The role of cytokines in the generation of inflammation and tissue damage in experimental gram-positive menigitis. J. Exp. Med. 171:439-448. 